AUDIT REPORT

\title{
UK National Audit of Early Syphilis Management. Case Notes Audit: Contact Tracing, Information giving, Follow-up and Outcomes
}

\author{
Hugo McClean FRCPI, David Daniels FRCP, Chris Carne MD FRCP, Paul Bunting MSc, \\ Rob Miller FRCP
}

on behalf of the National Audit Group of the British Association for Sexual Health and HIV

Summary: Contact tracing was provided for 683/781 (87\%, regional range 57-97\%) cases, and identified 997 traceable contacts of whom 511 (51\%) were seen, short of the recommended standard of $60 \%$. However, the performance range for this standard was $26-70 \%$, with 7 Regions achieving $60 \%$ or more. Two hundred and fifteen of 511 (42\%, range 3-73\%) contacts had syphilis. Treatment completion was recorded for 691 (88\%, range $71-100 \%)$ cases, and resolution of lesions for $348 / 469$ (74\%, range 40-96\%) cases. Nationally, 419/764 (55\%, range 37-70\%) cases were recorded as having a two dilution (fourfold) or greater decrease in non-treponemal test titre within 3-6 months after treatment; not achieving this titre decrease was mainly attributable to non-attendance for follow-up and failure of titre levels to fall. Follow-up of infectious syphilis in UK genitourinary medicine clinics is poor and falls far short of that recommended by National Guidelines. Only $16(2 \%)$ cases had follow-up at intervals approximating to 1, 2, 3, 6 \& 12 months, and only 312 (40\%, range 5-61\%) cases attended at least two follow-up visits. Only 17 (7\%) of all 236 oral treatments (including switches to oral treatment), and $33(27 \%)$ of 123 cases with HIV infection were recorded as designated annual follow-up. Further work is needed to determine factors that account for the wide variation between Regions in contact tracing and follow-up performance.

Keywords (MeSH): Syphilis, Medical Audit, Nursing Audit, Contact Tracing, Patient Education, Outcome Assessment (Health Care)

\section{Introduction}

This paper accompanies the report on the case note audit of diagnosis and treatment of early syphilis carried out in 2004 by the National Audit Group in response to increasing numbers of cases of infectious syphilis in the UK. ${ }^{1}$ 


\section{Final}

$19 / 07 / 05$

\section{Methods}

The Audit methodology and organisation is described in the report on diagnosis and treatment. ${ }^{1}$ This part of the Audit provides data that allows comparisons of practice in contact tracing, information giving, follow-up and outcomes to be made between Regions, as well as to national averages and National Guidelines. ${ }^{2}$ Data aggregated by NHS Trusts within Regions, not published in this paper, has been provided to Chairs of Regional Audit Groups to allow service comparisons between Trusts.

\section{Results}

\section{Contact tracing}

The case note audit asked two questions about contact tracing: "Was contact tracing carried out" and "If yes, with whom ...." Therefore, two measures of contact tracing could be calculated: the total number of cases provided with contact tracing, and the number having contact tracing provided by each healthcare profession. Table 1 shows performance based on both questions. Of 673 cases who were provided with contact tracing by any healthcare professional, 611(91\%) had contact tracing provided by health advisers. It is noteworthy that there is strong agreement between the rates of contact tracing provision obtained in both these questions. Contact tracing identified 997 traceable contacts, and the national ratio of traceable contacts to number of index cases was 1.28 (regional range 0.43 to 1.79). Table 1 shows the wide range of positive adult contact rates reported, although 8 of the 16 Regions had positive adult contact rates above $46 \%$ (see discussion).

\section{Information giving}

The following are the performances with regard to provision of the information items specified in the National Guidelines: ${ }^{2}$ partner notification: 657 (84\%, range 61-97); it is noteworthy that this figure agrees with the other two measures of contact tracing described above; health education: 545 (70\%, range 46-100); and past treatment: 373 (48\%, range 9-78\%). Only 45 (6\%, range $0-$ $13 \%)$ patients were provided with a letter documenting treatment.

\section{Follow-up}

Table 2 shows performance relating to follow-up. These results are based on a question that asked for specific follow-up intervals. The following number(s) of follow-up visits were recorded: no visits 179 (23\%, range 5-51\%); one visit 275 (35\%, range $15-66 \%)$; two visits $80(10 \%$, range 0 - 


\section{Final}

$20 \%)$; three visits $173(22 \%$, range 2-39\%); four visits 21 (3\%, range $0-8 \%)$; more than four visits 29 (4\%, range 0-12\%), and other non-specified follow-up 24 (3\%, range 0-8\%). There were 127 permutations of follow-up. Only 16 cases $(2 \%)$ had follow-up at intervals approximating to 1, 2, 3 , $6 \& 12$ months (the minimum follow-up schedule recommended by the National Guidelines ${ }^{2}$ ).

Table 3 shows the responses to an additional question about eventual follow-up outcomes. Only $36 \%$ of cases were ascribed the ideal outcome of a seronegative or serofast non-treponemal test (NTT) titre and discharge at one year, mainly explained by three other responses, failing to return for follow-up (34\%), lifetime annual follow (7\%), and other reasons (16\%). The following other reasons for not achieving this ideal outcome were as follows: follow-up at another centre (34 cases, 4\%); negative NTT or serofast at less than 12 months (20 cases, $3 \%$, of whom 3 cases were discharged); follow-up for HIV infection (15 cases, $2 \%$ ); continuing management (14 cases, $2 \%$ ); other specified non-attendance (11 cases, 1.4\%); annual follow-up for 5 years ( 6 cases, $0.8 \%$ ) - this outcome was reported from one Region only; moved away ( 5 cases, $0.6 \%$ ); negative NTT or serofast at 12 months but not discharged (2 cases, $0.3 \%$, one case received an oral treatment, and the other parenteral treatment, both cases had negative tests for HIV infection), and various other responses for $19(2 \%)$ cases. (See Discussion for further interpretation of this outcome).

Based on final treatments (after treatment switches), 189 of $236(80 \%)$ cases who received oral treatment and 414 of $513(81 \%)$ cases who received parenteral treatment attended for follow-up. Only 56 cases were designated as requiring annual follow-ups. Of these, $17(30 \%)$, including 9 cases with HIV infection, had received oral treatment. Of the remaining 39 parenteral treatments, $24(62 \%)$ were cases with HIV infection. Hence, annual follow-up was only justified for 41 (73\%) of these cases. Only $17(7 \%)$ of all 236 oral treatments (including switches to oral treatment) were designated annual follow-up. Only $33(27 \%)$ of 123 cases with HIV infection were recorded as having annual follow-up. Fifteen (4\%) of 410 completed parenteral treatments for cases not known to have HIV infection were designated annual follow-up

\section{National Guideline outcomes}

Table 4 shows outcome performance compared against specific standards in the National Guidelines, ${ }^{2}$ and against national average performances. A very wide range of resolution rates was recorded across the Regions. Nationally, 469 cases were designated as applicable for clinical 


\section{Final}

lesion resolution. Of these, 123 were oral treatments, 327 were parenteral treatments and one received both an oral and parenteral treatment, of whom 97(79\%), 250 (76\%) and none, respectively, were recorded as having lesion resolution. The remaining 18 cases comprised 8 cases treated elsewhere, 6 for whom the question about resolution was not answered, 3 not treated and one case for whom resolution was not known.

The low rate of recording a two dilution (fourfold) or greater decrease in NTT titre within 3-6 months after treatment was explained by the following outcomes: failed to re-attend $(152,19 \%)$; expected titre drop not attained $(117,15 \%)$; not tested $(26,3 \%)$; other reasons $(14,2 \%)$; negative or neat titres at diagnosis $(17,2 \%)$, and this question was not answered for $(36,5 \%)$ cases. Of the 117 cases not attaining an expected titre drop, 105 (90\%) had a follow-up visit at 3 months and 48 (41\%) cases had a follow-up visit at 6 months.

Recording of treatment completion also displayed a fairly wide performance range of about $30 \%$. Nationally, of the 236 oral treatments (including switches to oral treatment and one case treated with both an oral and parenteral treatment), 195 (83\%) were recorded as completed, and of the 513 parenteral treatments, 494 (96\%) were recorded as completed. Nationally, 90 (12\%) treatments were not recorded as completed, accounted for by the following: no documentation of treatment completion for $42(5 \%)$ cases, $13(1.7 \%)$ cases were not treated based on this measure (note that 14 cases were not treated based on recorded treatments ${ }^{1}$ ), 11 (1.4\%) cases were treated elsewhere and treatment completion could not be commented on, treatment not completed in $12(1.5 \%)$ cases, and this question was not answered for $12(1.5 \%)$ cases.

Nationally, 511 adult traceable contacts were recorded as having being seen (at an own or other centre), and the range of performance, based on the National Guidelines standard of $60 \%{ }^{2}$ for the proportion of traceable adults seen, was very wide at around $45 \%$. However, 7 Regions achieved this standard or greater. Of the 98 cases not provided with contact tracing, 31 traceable contacts were recorded for 15 index cases, of whom 6 contacts were seen (contacts seen/traceable contacts $=19 \%$ ). This contrasts with 966 traceable contacts (discounting one outlier with 60 contacts) recorded for 683 index cases for whom contact tracing was provided and where 505 contacts were seen (contacts seen/traceable contacts $=52 \%$ ).

The national ratio of number of traceable contacts seen to number of index cases was 0.66 (regional range 0.29 to 0.97 ). 


\section{Final}

$19 / 07 / 05$

\section{Discussion}

This Audit provides evidence that performance in contact tracing and follow-up of index cases with infectious syphilis in parts of the UK falls short of the recommendations in the National Guidelines. ${ }^{2}$ Both the National Guidelines contact tracing performance indicator and the adult positive contact rate were highly variable across the Regions. Achievement by 7 Regions of the National Guidelines contact tracing performance indicator supports the use of this standard. However, none of the aggregated performance rates for the London Regions achieved this standard (although 9 of the 23 participating London clinics did achieve this standard); the average performance for the London clinics against this standard was 44\%. In their paper on contact tracing standards for gonorrhoea and genital chlamydial infection, Low and Radcliffe concluded that "Standards set using expert opinion are unrealistic when compared to evidence of what is achievable. Evidence based methods should therefore be used to derive outcome standards..." 3 Might this also be appropriate for infectious syphilis?

Nationally, the proportion of adult contacts found to have syphilis was slightly below the rates found in previous studies referred to in the National Guidelines (42\% vs $46 \%$ and $60 \%$ ). ${ }^{4,5}$ However, 8 Regions achieved rates within or above the range 46-60\%. Good agreement between three questions used to measure contact tracing provision suggests that about $15 \%$ of cases were not provided with contact tracing. Very little is known about the clinic structures and processes in the UK that support good contact tracing outcomes. Also, improving contact tracing outcomes for infectious syphilis is an effectiveness issue in North America, ${ }^{6,7,8}$ although little has been published about this in the UK.

The very low rates of recording of a two dilution (fourfold) or greater titre decrease in NTT titres within 3-6 months after treatment was mainly attributable to patients not attending for follow-up and failure of titre levels to fall. As NTT titres may take longer to decline in early latent syphilis compared to primary and secondary syphilis, use of this interval may not be appropriate for early latent syphilis. ${ }^{9}$ However, exclusion of early latent cases increased overall performance against this standard by only $5 \%$ to $59 \%$. The other NTT titre outcome audited was a seronegative or serofast NTT titre and discharge after one year of follow-up. Table 3 shows performance recorded against this standard for all cases. However, performance against this outcome was really only applicable to 427 cases who had parenteral treatment and who were not known to have HIV infection: based on this denominator, 164 (38\%) of these cases met this standard, only a small increase in performance (this denominator also excludes 27 cases who were not treated, were 


\section{Final}

treated elsewhere or where data about treatment was not provided). On the other hand, 327 (42\%) cases received an oral treatment and/or had HIV infection, and would not therefore have been eligible for discharge after one year. However, meeting this standard was reported for 111 (34\%) of these cases, even though the criteria for discharge were not met.

Based on a range of measures, there appear to be highly variable follow-up rates between Regions. Approximately three quarters of index cases attended for follow-up, but only half this number attended for at least two visits. In contrast to the management of gonorrhoea and genital chlamydia, where reducing the number of return visits to clinics may be appropriate, follow-up for serological tests is an essential aspect of management for infectious syphilis. Additionally, this Audit suggests that annual follow-up is not usually provided to cases receiving oral treatment. It is likely that most cases with HIV infection will have continued with follow-up, although this seems to have been poorly documented.

\section{Conclusions and suggested areas for practice improvement/intervention}

Further study is needed of UK genitourinary medicine clinic service structures and processes, as well as patient factors, in order to determine factors accounting for wide ranges in contact tracing and follow-up performance. Any possible impact of the current problems in meeting patient demand for services in UK genitourinary medicine clinics ${ }^{10}$ on contact tracing and follow-up for cases of infectious syphilis requires urgent attention.

All patients with infectious syphilis should be provided with contact tracing. It is possible that immediate (e.g. dark ground microscopy) or other interventions to achieve early diagnosis may help to facilitate this. Finally, exploration of contact tracing methods to improve outcomes may be warranted in the UK.

Detailed data, aggregated by Region, are available on the BASHH website:

http://www.bashh.org/committees/nag/index.htm

Acknowledgements are made in the report on Diagnosis and Treatment $^{1}$

Correspondence to: Dr. Hugo McClean, Department of Genitourinary Medicine, Conifer House, West Hull Primary Care NHS Trust, Conifer House, 32-36 Prospect Street, Hull, HU2 8PX. E-mail: Hugo.McClean@whpct.nhs.uk 
1 McClean H, Daniels D, Carne C, Bunting P, Miller R on behalf of the National Audit Group of the British Association for Sexual Health and HIV. Case Notes Audit: UK National Audit of Early Syphilis Management - Diagnosis and Treatment. Int J STD \& AIDS 200_; _:

2 British Association for Sexual Health and HIV. Clinical Effectiveness Group. UK National Guidelines on the Management of Early Syphilis 2002 [ http://www.bashh.org/guidelines/2002/early\$final0502.pdf] (last accessed 1 May 2005)

3 Low N, Welch J, Radcliffe K. Developing national outcome standards for the management of gonorrhoea and genital chlamydia in genitourinary medicine clinics. Sex Transm Infect 2004;80:223-9

4 Schober PC, Gabriel G, White P, Felton WF, Thin RN. How infectious is syphilis? Br J Vener Dis 1983;59:217-9

5 Phaosovadi S, Snidvongs W, Tasanpradit O, et al. Treatment of sexual contacts of syphilitic pregnant women. J Med Assoc Thai 1989;72:132-7

6 Ogilvie G, Knowles L, Wong $\mathrm{E}$ et al. Incorporating a social networking approach to enhance contact tracing in a heterosexual outbreak of syphilis. Sex Transm Infect 2005;81:124-7

7 Brewer DD, Potterat JJ, Muth SQ et al. Randomized trial of supplementary interviewing techniques to enhance recall of sexual partners in contact interviews. Sex Transm Dis 2005;32:189-93

8 Michaud JM, Ellen J, Johnson SM, Rompalo A. Responding to a community outbreak of syphilis by targeting sex partner meeting location: an example of a risk-space intervention. Sex Transm Dis 2003;30:533-8

9 Romanowski B, Sutherland R, Fick GH, Mooney D, Love EJ. Serologic response to treatment of infectious syphilis. Ann Intern Med 1991;114:1005-9

10 Foley E, Patel R, Green N, Rowen D. Access to genitourinary medicine clinics in the United Kingdom. Sex Transm Infect 2001;77:12-4 
Table 1 Contact tracing performance in early syphilis ( $\mathrm{N}=781$ unless otherwise stated)

Contact tracing indicator Number (National average as \%) Regional ranges as \%

\begin{tabular}{lrr}
\hline Contact tracing provision & $683(87)$ & $57-97$ \\
Contact tracing provider & & \\
Health Adviser & $611(78)$ & $57-97$ \\
Doctor & $111(14)$ & $0-35$ \\
Nurse & $42(5)$ & $0-16$ \\
Other & $2(0.3)$ & $0-3$ \\
Not answered: & $108(14)$ & $3-43$ \\
$\quad$ Any healthcare provider & $673(86)$ & $57-97$ \\
Positive adult contacts/contacts seen $(n=511)^{\dagger}$ & $215(42)$ & $3-73$ \\
Children seen/traceable children $(n=13)$ & $12(92)$ & $0-100$ \\
Positive cases in children $(n=13)$ & $0(0)$ & Not applicable
\end{tabular}

${ }^{*}$ More than one choice of provider was used

†One outlier index with 60 contacts discounted

Table 2 Follow-up of cases with early syphilis $(\mathrm{N}=781)$

\begin{tabular}{lrr}
\multicolumn{1}{c}{ Follow-up indicator } & Number (National average as \%) & Regional ranges as \% \\
\hline Any follow-up & $604(77)$ & $49-95$ \\
At least two follow-up visits & $312(40)$ & $5-61$ \\
Follow-up during selected intervals & & \\
Up to 6 weeks & $230(29)$ & $9-57$ \\
7 weeks to 4 months & $328(42)$ & $10-67$ \\
$>$ 4 months to 9 months & $175(22)$ & $6-44$ \\
$>$ 9 months to 12 months & $182(23)$ & $0-37$ \\
$>12$ months & $32(4)$ & $0-12$ \\
$1,2,3$ months & $136(17)$ & $2-28$ \\
\hline
\end{tabular}


Table 3 Follow-up outcomes in early syphilis $(\mathrm{N}=781)$

Follow-up outcome* Number (National average as \%) Regional ranges as \%

VDRL/RPR negative/serofast at 1year after treatment, patient discharged ${ }^{\dagger}$

$276(36)$

$13-56$

Re-infected/retreated

$0-6$

Clinical relapse, retreated

Failed to return

$262(34)$

16-55

Treatment failed, retreated

$10(1)$

Annual follow-ups for life

Negative initial tests repeated at 3 months

Other

Not answered

$15(2)$

$0-10$

${ }^{*}$ More than one choice of follow-up outcome was used

† Denominator excludes 15 cases with negative initial tests repeated at 3 months

Table 4 Performance against outcome measures listed by Clinical Effectiveness Group for early syphilis management ( $\mathrm{N}=781$ unless otherwise stated)

\begin{tabular}{|c|c|c|c|}
\hline Outcome measure & Standard & $\begin{array}{l}\text { Number (National } \\
\text { average as \%) }\end{array}$ & $\begin{array}{c}\text { Regional ranges as } \\
\%\end{array}$ \\
\hline Resolution of clinical lesions & Not specified & & \\
\hline All stages* $\left(n=469^{\dagger}\right)$ & & $348(74)$ & $40-96$ \\
\hline Primary syphilis $\left(n=231^{\dagger}\right)$ & & $176(76)$ & $33-100$ \\
\hline Secondary syphilis $\left(n=193^{\dagger}\right)$ & & $149(77)$ & $12-100$ \\
\hline $\begin{array}{l}\text { Two dilution (fourfold) or greater decrease in VDRL/RPR } \\
\text { titre within 3-6 months after treatment }\left(n=764^{\ddagger}\right)\end{array}$ & Not specified & $419(55)$ & $37-70$ \\
\hline Completion of treatment & $95 \%$ & $691(88)$ & $71-100$ \\
\hline Adult contacts seen/traceable adult contacts $(n=997) \S$ & $60 \%$ & $511(51)$ & $26-70$ \\
\hline
\end{tabular}

END. 